\title{
Chaos/Complexity Theory in Language Learning: An Ideological Look
}

\author{
Nima Shakouri \\ Roudbar Branch, Islamic Azad University, Iran \\ Morteza Teimourtash \\ Islamic Azad University, Science and Research Branch, Tehran, Iran \\ Mojtaba Teimourtash \\ Islamic Azad University, Science and Research Branch, Tehran, Iran
}

\begin{abstract}
Traditionally the process of learning is assumed to be a linear process; there exists a cause-andeffect, logical, and deterministic view of the world's system. In science and scientific realm, we are searching for the cause-and-effect connections while in chaos/complexity theory such a connection is not that much straightforward, as it seems to be unpredictable. Chaos/complexity scientists are interested in how disorder gives way to order in order to find, at least, one mind map for every single phenomenon, and how complexity arises in nature. With the advances in science, especially in meteorology, we have movements towards more uncertainty and unpredictability (Larsen-Freeman, 2002). The authors in the present paper provide some evidence to support that there are many striking similarities between chaos/complexity theory and language learning. In fact, chaos/complexity theory can be used as a metaphor in language learning, but more importantly it can replace the discourses which dominate and inform much of our current practice (Mallows, 2002). As Gleick (1987) holds the theory is a science rather than state, of becoming rather than being. In this paper, we will tend to outline the main ideas behind this theory, and relate them to language learning.
\end{abstract}

Index Terms - chaos theory, complexity, dynamicity of language, interlanguage

\section{INTRODUCTION}

Chaos theory and the studies on complexity have been influencing many different research fields, including Applied Linguistics. The emergence of the notion of chaos/complexity is rooted in the Big Bang era when the systematic nature of the universe was founded out of a chaotic act of God. In many religious books, the issue has been touched upon in a neat fashion. In the holy Quran, it is mentioned this way:

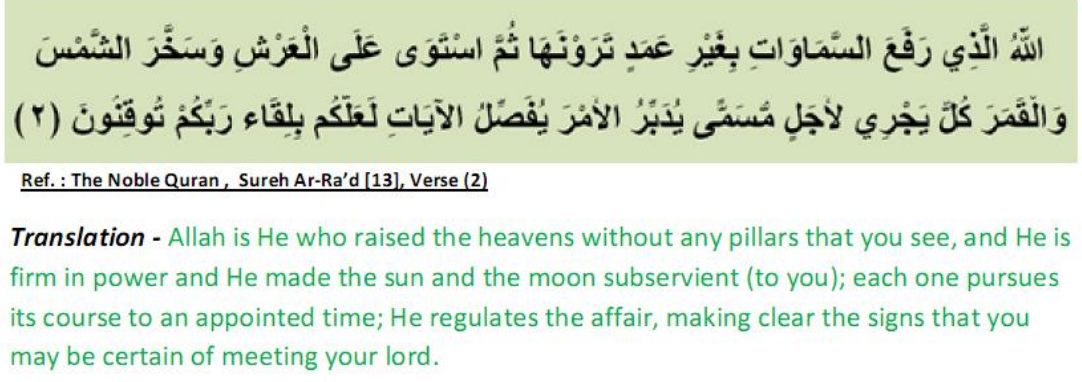

If one wants to trace the survived documents, what is taken for granted is that the notion of chaos/complexity is dated back to 1960s, when Edward Lorenz, a meteorologist, noticed that a very small difference could lead to large changes in the weather which make long-distant weather forecasts impossible to predict. The phenomenon which is the cornerstone of chaos theory is known as sensitivity to initial conditions. It holds that a small change in the initial conditions may bring about a dramatic change in the long-term behavior of a system (de Bot, 2008 cited in LarsenFreeman \& Cameron, 2008). Later, the concept has been applied to other areas of study like physics, mathematics, chemistry, and even sports. Regarding the importance of chaos/complexity theory, McAndrew (1997) implies that no one can find any reality without chaos. In effect, the popularity of studying complexity is fast becoming a new fad in the intellectual scene. In different fields of study, there is somehow an interest in the use of computation and other means, in order to study any complex phenomena. Then, what are the promises in such enticing fad about studying complex phenomena?

\section{LITERATURE REVIEW}




\section{A. Chaos/Complexity Theory}

The notion of chaos/complexity science, according to some scholars is a paradoxical notion. Larsen-Freeman (1997) believes that the two portions of chaos/complexity and science do not go together as the concept bear a contradictory paradoxical connotation. The term science is meant to convey any orderly systematic and complex body of knowledge within which each embodying element could be traced to its source of occurrence. Hence, chaos/complexity does the other way round. The connections exist within and among elements in a chaotic system are not traceable in a scientific fashion. In simple words, there exists a dynamic connection which cannot be determined or stated straightforwardly. To better appreciate the concept of complexity, let us first, elaborate on the significant features of it: (1) complexity involves interconnectivity; (2) complexity is not necessarily random; (3) complexity is unpredictable; (4) complexity is not arbitrary; (5) complexity involves sensitivity to an initial change; and (6) complexity involves dynamicity.

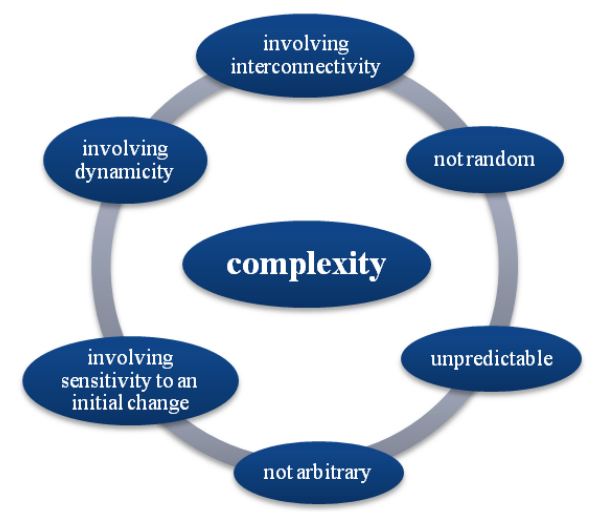

Larsen-Freeman (2000) sees complexity as "a metaphorical lens through which diverse perspectives can be accommodated, indeed integrated" (p.173). In order to spotlight the true sense over the concept of complexity to its real depth, some related issues should be highlighted here. The term complexity is usually mistaken with complicatedness, whereas the connotations are different or even divergent. Once a system is complex, it is for sure not independent, while complicatedness connotes not being simple.

To better appreciate the concept of interconnectivity in a complex system, let us delve into the issue first. In a complex system, there are numerous independent elements that are continuously interacting with each other, thereby simultaneously organizing themselves into a more multifaceted system (Harshbarger, 2007). According to McAndrew (1997, cited in Alemi \& Daftarifard, 2011), something is complex if a great many independent agents are interacting with each other in many ways, the richness of these interactions allowing the system as a whole to undergo spontaneous self-organization. In fact, the agents in the system are all the components of that system. These agents interact and connect with each other in unpredictable and unplanned ways. But from this mass of interactions, regularities emerge and start to form a pattern which feeds back on the system and informs the interactions of the agents. For example, in an ecosystem if a virus starts to deplete one species, this results in a greater or lesser food supply for others in the system which affects their behavior and their numbers. A period of flux occurs in all the populations in the system until a new balance is established.

There is one important point to be taken into consideration, and that is the notion of interacting which implies a very organized and understandable event following a preplanned designed, but the point here is that in a complex, or in a multifaceted system, there is no preplanned interaction. The notion of multifaceted system can be planned and predicted, but the triple effect of changing one connection to other connections' feedback is hard to estimate; in fact, this feature would make complexity distinguishable from complicatedness.

Secondly, being complex does not necessitate being random, where being random is the property of a variable which possesses a range of probable distribution of occurrences. Sometimes, being random may indicate a process which entails an unpredicted outcome far from the probability distribution. Either case could never ever necessitate equating randomness and complexity. This randomness is unpredictable and irregular in the sense that the time of occurrence is not known to us (Alemi \& Daftarifard, 2011). It also seems that a complex system, sometimes considered as a system with implicit principles, is not known to the audience except to its creator, whereas nothing is accidental in nature. The focus in randomness is mostly on the product phase while complexity is highlighting the process phase, both the process of building a phenomenon and the process of its analysis. There is the common ground that the chaos existing in the process layer of complexity might lose its peculiarity through the passage of time. This is mainly because noble entities do follow a chaotic fashion. Chaotic fashion to the extent that the noble entities are new ones and through the elapse of time, their chaotic fashion would be discovered and known to everyone. Along the same line, in the holy Quran, it is stated that: 


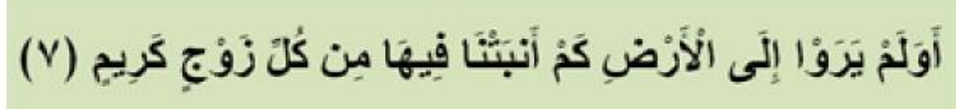

Ref. : The Noble Ouran. Sureh Ash-Shu'ara' [26], Verse (7)

\section{Translation - Do they not see the earth, how many of every noble kind we have caused to grow in it?}

The unpredictability issue may have cumulative effect and results in a sudden highly-organized system. This is in no way an indication that complexity is arbitrary, because as the concept of complexity could be well-defined regarding its units, changes of state, interactions, and surroundings, so being arbitrary does not fit the definition of a complex system. It is worth emphasizing that the non-arbitrary nature of a complex system is for sure sensitive to the feedback it receives, while the output of a subsystem is utilized as the input of another subsystem in a chain reaction mode of occurrence. Many scholars in the literature specify a certain form of emergence of a major overall effect from a slight initial change as a common characteristic of complexity models in general (Baofu, 2007). The sensitivity to initial conditions, according to Harshbarger (2007), means that small variations inherent in a complex system at a given time can result in large differences in the system's behavior over time. Alemi and Daftarifard (2011) insist "Edward Lorenz's (1993) butterfly metaphor in chaos/complexity indicates the importance of minor changes which lead to great changes at the end" (p. 37).

What deems important here to pinpoint is that the nature of the universe and whatever happens across the globe is indicative of a dynamic interconnected entity. The events happening every now and then are interconnected and ongoing. So there is no at the end position for any event. Any end position is a new initial condition for another event. Ahmadi (2011) in line with the sensitivity to initial conditions in chaos theory argues, "a small and seemingly unimportant initial condition may create a big and unexpected outcome" (p. 296). Along the same line, Gleick (1987) further states "tiny differences in input could quickly become overwhelming differences in output" (p. 8). It could be concluded in this way that, due to the potentiality of the input elements which will or will not be triggered because of the randomness of their nature, our predictions of the output of a chaotic system may be inexact and uncertain, for it might follow a binary fashion of zero and one mode; that is, at the most probable moment of possibility in a chaotic system, it turns out to give out a reverse output, and that is exactly due to the randomness in the behavior of the chaotic system.

\section{B. Dynamic/Chaotic System and Language}

To Larson-Freeman (1997) language is a complex nonlinear system. It is dynamic in nature because it changes overtime, or it may change overtime because it is dynamic in nature. In fact, the term dynamic equates growth and change. Dynamicity involves non-linearity. It is nonlinear because there is no one-to-one correspondence between form and function. Also it is dynamic in the sense that there is no difference between the current use and change/growth of language; they are the same. Messages emerge as the result of speaker and listener's collaborative effort. Alemi and Daftaifard (2011) maintain fractality of language results in the existence of infinite number of behavior within a finite system.

The dynamicity of language is mostly due to the dynamicity of its users. Human beings have been proving, during the long course of history, their brilliant feature of creativity (or smart applications) which makes them distinguishable from other creatures. Since language and its users are, both, dynamic phenomenon in nature, consequently the result is a weird dynamic system but at the same time, chaotic one, namely as language system. In sum, any system in the universe has got a counter system which acts as the complementary portion of the process of the chaotic phenomenon. This notion is prevalent in each and every master system across the globe. In religious books, such notion has been highlighted in a neat fashion that for instance the sun and the moon, or day and night are not competing each other but interacting in a chaotic fashion. In the holy Quran, such systematic maneuverability of chaotic phenomenon is spotlighted this way:

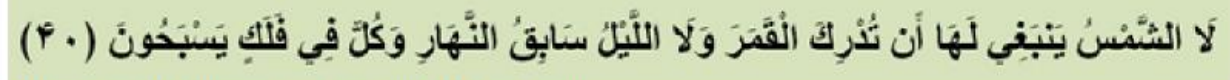

Ref. : The Noble Quran, Sureh Ya-Sin [36], Verse (40)

\section{Translation - Neither is it allowable to the sun that it should overtake the moon, nor can the night outstrip the day; and all float on in a sphere.}

Traditionally, it was considered true that by being exposed to the forms of one language, the linguistic competence was triggered, so it is easy to learn, or even acquire, that language. However, due to the dynamic feature of language and human beings, the social, and strategic competences are introduced. Then there exists a covering notion of nurture 
which embodies all the above mentioned issues interconnectedly. In a sense, the deficiency or poor performance in one variable is compensated by other adjacent variables.

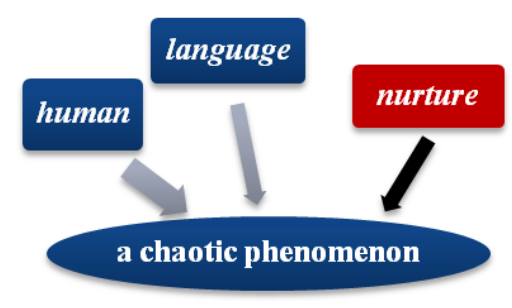

Feignbaum (1978, cited in Thietart \& Forgues, 1995) shows that a system passes from a stable to a periodic and from a periodic to a chaotic situation when the strength of the link between variables changes. In fact, a dynamic system is a system whose time-dependent variables are non-linear.

Another characteristic of dynamic systems, according to Thetart and Paiva (1995), is that it is deemed to be as a dissipative system; that is, such a system dissipates its energy. The chaotic evolution may get organized around structures that we find at different scales, named the strange attractors. A new form of order is found out of chaos. The apparent random behavior gets attracted to a given space and remains within its limits. These attractors were first called strange because of their unexpected and strangely regular shape, such as the butterfly wing shaped attractor of Lorenz. Winter (1994, cited in Larsen-Freeman, 1997) argues that all information systems used to be fractal in shape, in order to make them comprehensible and thus shareable. In fact, the attractor creates an implicit order within chaos. Inside the attractor space, the system behavior is highly complex and unstable (Thietart \& Forgues, 1995).

\section{Nurture in Dynamic/Chaotic System}

Schneider and Somers (2006) maintain, "dissipative structures may react disproportionately to an environmental change" (p. 356). A small exogenous event may trigger a change in the fundamental character of a system. The phenomenon of large, disproportionate change is referred to, as mentioned earlier, as the butterfly effect. Accordingly, properties of the system may emerge from its parts, rather than being imposed by the environment (Holland, 1998). The butterfly effect could be deemed compatible to the language learning environment, classroom settings for instance, through which the dynamicity of language learning phenomenon is spotlighted. Here the notion of nurture in language learning realm is highlighted as one of the fundamental elements that can affect the rate of dynamicity, in other words, chaoticity, in every linear or non-linear system. This notion provides the language learning realm with more dimensions in which interconnectivity in learning system is strengthen.

The notion of nurture, in simple terms, context, is known as the turning point for each and every system in order to manifest its rate of dynamicity. This is mainly because, as mentioned above, every system is dynamic in nature, but it is not true in every nurture domain, whereas the appropriate nurture lets a system activate its own features of dynamicity in its own trend of occurrence.

Aside from all the justifications above, dynamic systems are attracted to paths that can be traced in the two axes of time and space. It could be highlighted here that a complex system does not follow a predictable pattern; that is, the reaction and reflection of a chaotic system would vary in various time and space. Along the same line, Larsen-Freeman (1997) insists that a complex nonlinear system does have a strange attractor in the sense that there dominates cycle which repeats itself, but at the same time, there are no identical cycles which follow the same path of development. The point to be emphasized here is that along the course of the development, a chaotic system may shows a distinguished pace of modification, which is in turn identical and unique.

\section{Chaos/Complexity Theory and Language Learning}

Chaos/complexity theory offers a lens for anti-reductionist thinking that language itself is an aggregation of static units or products which are combined in speech in a dynamic process (Mallows, 2002). Accordingly, Mallows (2002) argues:

The dynamic patterns of complex systems display feedback loops. There's nothing driving that system since the dynamics come from within the system itself, and the system uses feedback to move on, to evolve and to develop. These complex systems are adaptive; they take in external influences, and change their internal structure to take advantage of the new circumstances. (p. 5)

The existence of such feedback loops provides the opportunity of having a dynamic system. In language learning realm, such loops could not have been designed due to the high rate of changing factors of either affecting or affected ones. 


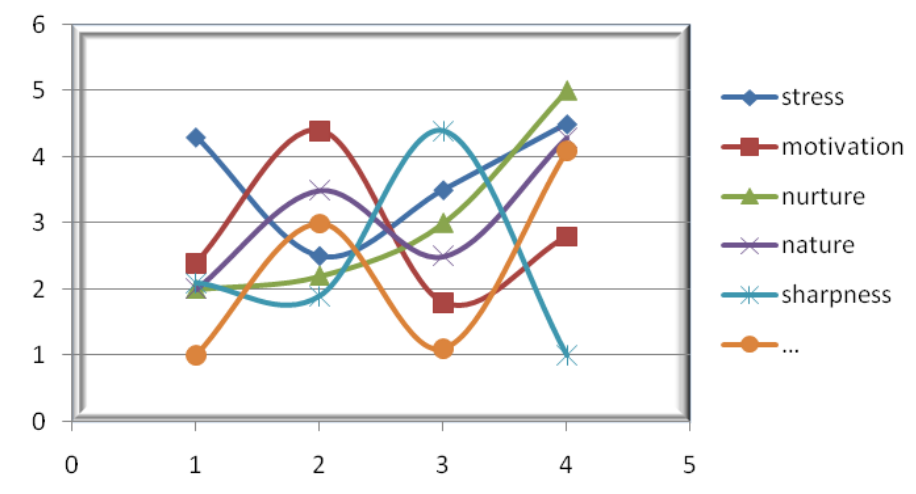

It is worth mentioning that all facets in every segment are interconnected with each other; in fact, any degree of change in one, due to any cause, would alter the proper manifestation of the output. No matter how these facets are connected to each other, or how much the degree of the changes are, the result, as the consequences of the butterfly effect mentioned above, is hard to estimate, or predict to some extent.

Larsen-Freeman (1997) also insists that these feedback loops accepted as agents of biological evolution can be applied to learning. Whereas, in biology, the feedback is provided by natural selection, and the improvement of the models is called evolution, in cognition the feedback comes from teachers and direct experience, and the improvement called learning.

As pointed by van Lier (1996), "we can neither claim that learning is caused by environmental stimuli (the behaviorist position) nor that it is genetically determined (the innatist position) (p. 170). It is worth regarding the classroom as a complex adaptive system in which "it is fruitless to search for causal relations" (van Lier, 1996, p. 38). In fact, "learning is the result of complex (and contingent) interactions between individual and environment." (van Lier, 1996, p. 170). It goes without saying that there seems to be much in common between language and complex non-linear systems. Languages go through periods of chaos-and-order, as do other living systems (Larsen-Freeman, 1997). Moreover, language growth occurs at the border between these two - chaos and order — which has been termed the edge of chaos by Waldrop (1994). As Larsen-Freeman (1997) puts it, languages undergo non-linear changes diachronically. New forms, for instance, enter and leave the language in a no-additive and non-predictable way (HadidiTamjid, 2007). Indeed, different speakers may use different forms to mean the same thing. The best thing we can do is to explain a change after its occurrence, without making exact predictions of what that change will occur next.

\section{E. Dynamicity of Language}

Finch (2002) argues that language acquisition can be said to be chaotic process if it is non-linear, iterative, and sensitive to initial conditions. The concept of non-linearity implies that the output is disproportionate to its input (Mallows, 2002); there exists no cause-and-effect relation between these two actions. It is hard to say that there is no sign of cause-and-effect between the input and the output, it is better to put it in this way that, there is no fixed and linear cause-and-effect relation between input and output. In other words, as the nature of causing elements is dynamic, the results and also the process of approaching the results will definitely be dynamic.

By iterative, we mean the learning output becomes the input of the next cycle. The concept of sensitivity to initial conditions also proposes that minor changes at a process may result in different and unpredictable outcomes. However the noticeable point here is that, one single input is not triggered at one moment, and then waits for obtaining the output, desired or otherwise. In fact, a wide range of inputs will, simultaneously, be triggered in a fraction of a second, and a wide range of outputs will be sensed consequently. Hence, no one may be able to make the slightest image of such bunch of inputs and outputs in order to make a fixed paradigm for them.

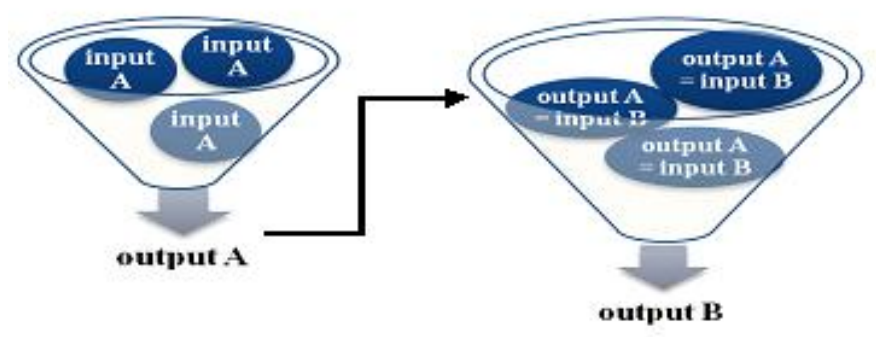

As is true of other dynamic non-linear systems, language satisfies both the criteria of complexity and dynamicity. It is complex since it is composed of various subsystems: phonology, morphology, syntax, semantic, pragmatics, and all of which are interdependent. A change in any one of them can result in change in the others (Larsen-Freeman, 1997). In 
other words, the behavior of the whole results from the interactions of the subparts.

Complex nonlinear systems exhibit sensitive dependence on their initial conditions, and language is no exception (Larsen-Freeman, 1997). The initial conditions might be called Universal Grammar which contains a set of principles that apply to all languages and whose function is to constrain the shape of language. These principles have a huge impact on defining the 'strange attractor' of human language (Larsen-Freeman, 1997). Hadidi-Tamjid (2008) believes that complexity is a two-folded concept. It is related to the fact that "language is a collection of static units but their use in actual speech involves an active process" (p. 11).

By dynamicity, as mentioned earlier, we mean a nonlinear system in which everything is interconnected. Also the system is dynamic in the sense that it changes by time. In fact, language is not a static object, but a system which is in constant movement and its interacting elements influence and is influenced by each other. As language is in evolution, so is learning. Phonological systems tend to change systematically but unpredictably. Contact with other languages can bring about catastrophic changes in syntax. For example, the loss of case endings on nouns in Old English is thought to be due to contact with Old Norse (Lightfoot, 1999, cited in Mitchener \& Nowak, 2004, p. 701). Michener and Nowak (2004) argue, "language change is unpredictable and highly sensitive to perturbations". (p. 703) Many changes, particularly those associated with borrowed vocabulary, are triggered by language contact. Larsen-Freeman (1997) argues, "The changes which language undergo diachronically are nonlinear" (p. 147). She also adds, "New forms enter and leave the language in a non-incremental fashion" (p.147). Changes in languages are isomorphic (Larsen-Freeman, 1997).

Larsen freeman (1997) claims, "every time language is used, it changes" (p. 148). Moreover, that the user's grammar is changed may lead to change at the global level. The act of using the language meaningfully has a way of changing the grammar system in the user (Diller, 1995, cited in Larsen-Freeman, 1997, p. 147). Besides, as the user's grammar is changed; this would set in motion a process which may lead to change at the global level.

In sum, the concept of dynamic as applied to language refers to the fact that language can be described as an aggregation of static units or products, but their use in actual speech involves an active process, usually referred to as parole or performance (Larsen-Freeman, 1997). Gleick (1987) holds that one of the major tenets of complexity theory is that it is a science of process rather than state, of becoming rather than being.

\section{F. Culture: The Milestone of Dynamicity}

Regarding the issue of dynamicity of chaotic systems, there are a mass of variables affecting the output of a system. Since language realm is the one which best fits the chaos/complexity theory and is regarded as a dynamic system, there are a number of elements affecting its dynamic nature, namely as the notions of nurture, typology of language, and human beings as the major decisive and pivotal element. Once the dynamic nature of language as a chaotic system is involved in the process of learning a language, the elements affecting the dynamicity is multiplied by the seconds of engagement in the process. As a result, there are multitude of factors, important of which is the issue of culture. Culture is the common ground for the dynamicity of language as the system of system and at the same time, human beings as the most unpredictable creatures of God. The unpredictability of human beings is rooted in the major distinction between human beings and other creatures, i.e. mind.

Culture is the manifestation of the development of mind, and its importance is so high that learning a new language is learning a new culture. Culture is what a group of people think, and how they think; because of that, some scholars believe in the equilibrium of thought and culture because culture is what people think. These issues are in line with one another and in turn would result in a major homogeneous phenomenon. That is to say, the dynamicity of mind would strengthen the dynamicity of culture, whereas the dynamicity of culture would be decisive and in direct proportion to the dynamicity of language as a complex chaotic system. There is one more point in this regard worth knowing. Being dynamic is known as the source of any change, and is an undeniable rule for all creatures all over the world, but what is eye-catching about them is the pace of change, which is just different among them. This is exactly the border line of the notion of creativity in language learning which is human-specific and is manifested through the application of culture. Furthermore, it should be noted here that the notion of culture is a dynamic notion. It might be quite true that through the axis of time, there is not static entity in the world. All entities across the globe are dynamic. In the same lane, the holy Quran has stated that even mountains could not be considered as solid and static. It is mentioned:

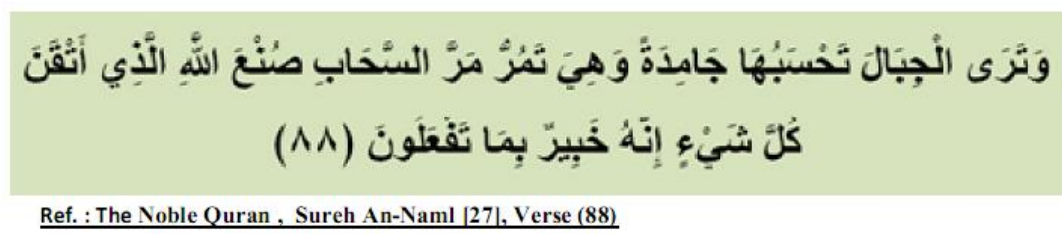

Translation - And you see the mountains, you think them to be solid, and they shall pass away as the passing away of the cloud-the handiwork of Allah who has made everything thoroughly; surely $\mathrm{He}$ is aware of what you do. 


\section{G. Chaos/Complexity Theory and Interlanguage}

Through the process of learning a new language, and out of chaos existing in the whole process, a new language would emerge, namely as the interlanguage, which oscillates between the first and second language as the main attractors present at the scene of consolidation. In the course of the development of the interlanguage, there happens a tune-in game of adjusting the attractors. An attractor is "a region of a system into which the system tends to move" (Larsen \& Cameron, 2008 , p.50). Once the movement is towards one of the two attractors namely as the first and second language, there is not always attraction at work. Sometimes the repulsion force wins the game and would in turn make a better adjustment, a better adjustment regarding the appropriateness and promptness. The chaotic nature of language paves the way to restructure the interlanguage system in the long process of restoration. Due to marching the self-organizing rhythm of chaotic system, along with the prevalent sensitivity to initial condition, the interlanguage system would manifest the whole matrix into a tuned language, and this is mainly because the interlanguage system is "feedback sensitive" (HadidiTamjid, 2008). The feedback received by the chaotic interlanguage system would be processed both as the initial condition of a strange attractor, and at the same time, as the follow-up feedback of a developing system.

One more point to be brought to great consideration is that, the idea that the strange attractors affect the interlanguage system is quite right. But what count here is that not all strange attractors belong to the target language domain. According to Larsen Freeman (1997), there is a plethora of similarities in the interlanguages of speakers with different L1, but the points of departure lies in the strange attractors of their L1s. This could be in turn a good example of the nonlinearity of the interlanguage system.

Mallow (2002) argues, "there are many interacting factors at play which determine the trajectory of the developing interlanguage" (p. 5). Of course, no one of these factors, according to Larsen-Freeman (1997), by itself is a determining factor, the interaction of which, however, has a very profound effect. There is an interaction between the language forms in a learner's system: when a learner starts to learn a new form, formerly mastered forms will become destabilized (Mallow, 2002).

A learner's interlanguage is also self-referencing in that it grows organically. While it can to a certain extent be described by rules, it is not produced by them. It is constantly changing and reacting to the feedback it receives; it is an open system, and moves to the strange attractor which gives it both impetus and order. Indeed, the phenomenon of fossilization, probably, occurs as a result of the interlanguage becoming closed, and setting to a fixed-point attractor (Mallow, 2002).

\section{CONCLUSION}

Like any other revolution, chaos/complexity is a reaction to incapability of isolationist methodology of doing research. In the same vein, as what Larsen Freeman(2002) claims, to better digest the existing chaos/complexity agenda, having a reductionist view or even a mere holistic perspective would not suffice. There should exist an amalgamation of these two perspectives in a fuzzy fashion. The authors in the present paper believe that having a discourse-based rationale on understanding the complexity of language learning will contribute teachers to have a thorough comprehensive understanding which is conducive to the process of learning. As the final remake which is a new reformulation of what was implicitly mentioned in the present article, the authors aimed at the idea that chaos/complexity is the backbone of systems which are not homeostatic in nature, namely as open system (Larsen-Freeman, 2002). Language as an open system is dynamic in its existence and the presence of dynamicity in the whole process of learning and tuning such system would in turn raise the complexity level which would renders language as a more chaotic entity.

\section{REFERENCES}

[1] Ahmadi, A. (2011). Chaos theory and language assessment: The effect of sensitivity to initial conditions on test performance. International Journal of Humanities and Social science, 1(17), 293-296.

[2] Alemi, M., Daftarifard, P., \& Patrut, B. (2011). The implication of chaos/complexity theory into second language acquisition. BRAIN. Broad Research in Artificial Intelligence and Neuroscience, 2(2), 34-40.

[3] Allah All-Mighty (n.d.). The Noble Qur'an. www.quraneasyurdu.net/ 100036/intercession/p.html .

[4] Baofu, P. (2007). The future of complexity: Conceiving a better way to understand order and chaos. London: World Scientific Publishing Co.

[5] Finch, A. (2002). A systems view of the EFL class: Mapping complexity. Retrieved in 2012, from http://www.finchpark.com/arts/index.html.

[6] Gleick, J. (1987). Chaos: Making a new science. Viking Penguin Inc. London.

[7] Hadidi-Tamjid, N. (2007). Chaos/complexity theory in second language acquisition. Novitas-ROYAL, 1(1), 10-17.

[8] Harshbarger, B. (2007). Chaos, complexity and language learning. ICU Language Research Bulletin, 22, 17-31.

[9] Holland, J. H. (1998). Emergence: From chaos to order. Reading, Massachusetts: Addison Wesley.

[10] Larsen-Freeman, D. (1997). Chaos/complexity science and second language acquisition. Applied Linguistics18(2), 141-65.

[11] Larsen-Freeman, D. (2000). Techniques and principles in language learning ( $2^{\text {nd }} \mathrm{ed}$.). Oxford: Oxford University Press.

[12] Larsen-Freeman, D. (2002). Language acquisition and language use from a chaos/complexity theory perspective. In C. Kramsch (Ed.), Language acquisition and language socialization: Ecological perspectives (pp. 33-46). London: Continuum.

[13] Larsen-Freeman, D., \& Cameron, L. (2008). Complex systems and applied linguistics. Oxford: Oxford University Press.

[14] Mallows, D. (2002). Non-linearly and the observed lesson. ELT Journal 56(1), 3-10. 
[15] McAndrew, D. (1997). Chaos, complexity, and fuzziness: Science looks at teaching English. English Journal, 86, 37-43.

[16] Mitchener, W. G., \& Nowak, M. A. (2004). Chaos and language. Proceedings of the Royal Society of London, Biological Sciences, 271(1540), 701-704, April. DOI 10.1098/rspb.2003.2643.

[17] Schneider, M., \& Somers, M. (2006). Organizations as complex adaptive systems: Implications of complexity theory for leadership research. Leadership Quarterly, ISSN 1048-9843, 17 (4), 351-365.

[18] Thietart, R.A., \& Forgues, B (1995). Chaos theory and organization. Organization Science, 6(1), 19-31. Retrieved in 2012, May from www.jstor.org.

[19] van Lier, L. (1996). Interaction in the language curriculum: Awareness, autonomy, and authenticity. London: Longman.

[20] Waldrop, M. M. (1994). Complexity: The emerging science at the edge of order and chaos. London: Penguin.

Nima Shakouri is currently a Ph.D. candidate of TEFL at Islamic Azad University, Science and Research Branch, Tehran, Iran. He has taught English courses for over a decade at different universities. Moreover, he has published nationally and internationally.

Morteza Teimourtash is currently a Ph.D. candidate of TEFL at Islamic Azad University, Science and Research Branch, Tehran, Iran. He is an English instructor.

Mojtaba Teimourtash is a holder of MA in TEFL. He has studied at Islamic Azad University, Iran. He is an English instructor. 